\title{
Ternary Kinetic Models for Rational Design of Molecular Glues
}

\author{
Eugene F. Douglass Jr.; ${ }^{1, *}$ Chad J. Miller ${ }^{2}$
}

\author{
${ }^{1}$ Pharmaceutical and Biomedical Sciences, University of Georgia, 250 W. Green Street, Athens, GA 30602, \\ USA. \\ ${ }^{2}$ Department of Biochemistry \& Institute for Protein Design, University of Washington, Seattle, WA \\ 98195, USA. \\ *corresponding author (eugene.douglass@uga.edu)
}




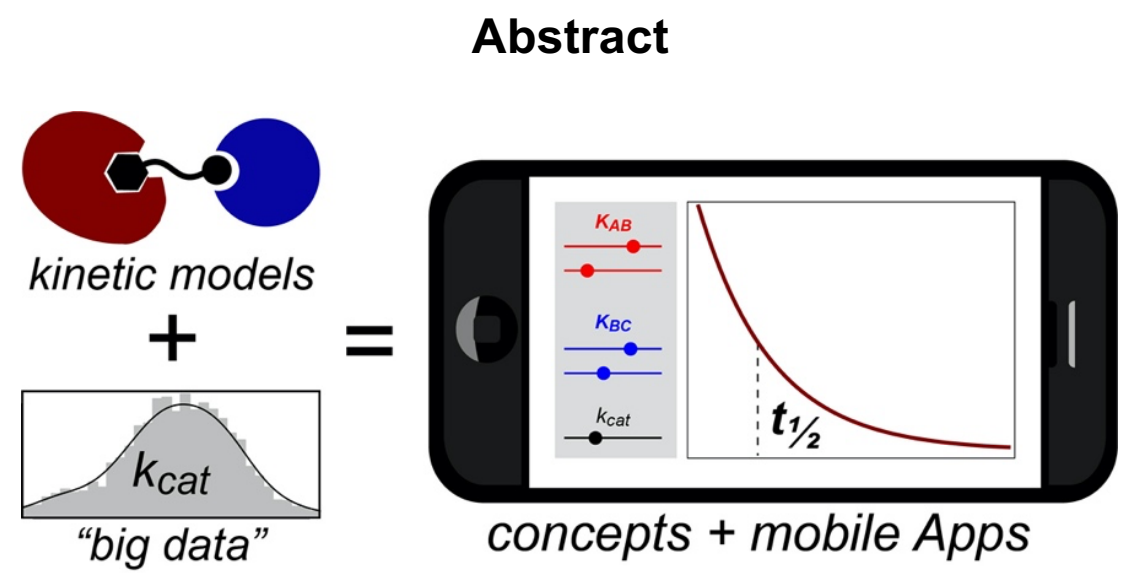

Recently, academic and industrial interest in molecular glue-based therapeutics has grown dramatically. Traditional drugs are designed to act on single targets, whereas molecular glues simultaneously bind two targets. By forming a ternary complex, molecular glues can create new therapeutic effects, such as rewiring cellular machinery to degrade specific proteins. Unfortunately, rational design of these therapies is challenging as current pharmacological theory is based on binary complex equilibria.

Here, we extend our previous ternary-complex equilibrium work (JACS, 2013, 135, 6092) to derive a set of kinetic models highly analogous to Michaelis-Menten kinetics. This similarity has enabled us to derive conceptual rules-of-thumb and identify the weakest binding affinity as the most important engineerable parameter in the design of ternary-complex-based therapeutics. Finally, we have combined these equations with "big data" from new thermodynamic and kinetic databases to build interactive online tools that enable non-computational investigators to graphically simulate their own systems:

- https://douglasslab.com/ternary equilibrium/

- https://douglasslab.com/Btmax kinetics/ 
MOLECULAR GLUES IN THE CLINIC

\section{Introduction}

Most clinical drugs are designed to bind a single target to form a binary complex (Figure 1a). These drugs typically act as inhibitors, blocking the function of their protein targets. In contrast, drugs that bind two targets - forming a ternary complex (Figure 1b) - have the potential to create new functions by scaffolding new protein protein-protein interactions (PPI). Classical examples of ternary-complexbased drugs include several biologicals such as heparin, interferon, growth hormones and monoclonal antibodies..$^{1-6}$ In addition, the efficacy of several natural and synthetic small molecules has been shown to be dependent on the formation of a ternary complex including: rapamycin, cyclosporine and lenalidomide. ${ }^{7}$

Recently, interest in rational design of ternary complex-based drugs has increased significantly. ${ }^{7,8}$ Prominent examples include both biological and chemical agents such as bispecific antibodies (BsAbs) and proteolysis targeting chimera's (PROTACs), respectively. ${ }^{4,9}$ BsAbs can simultaneously bind the CD3 receptor of T-cells and tumor antigens on cancer cells; this colocalization directs anticancer immune responses in the absence of classical T-cell activation or TCR specificity. PROTACs simultaneously bind E3 ubiquitin ligases and oncogenic proteins, causing ubiquitination and degradation of pathogenic proteins. BsAbs first entered the clinic in 2009, and now over 40 different bsAb's are currently in clinical development for various cancer indications. ${ }^{4,10}$ In 2019, PROTACs began initial clinical validation, and it has been estimated that 15 new PROTAC-based clinical trials will begin by the end of $2021 .{ }^{11}$

\section{CURRENT PHARMACODYNAMIC THEORY}

Current pharmacodynamic theory is built off of the Langmuir Hill equation, which gives the fraction drug bound as a function of the total drug concentration $\left([\mathrm{Drug}]_{t}\right)$ and the binding affinity or dissociation constant $\left(K_{d}\right)$ for its molecular target: ${ }^{12-14}$

$$
\% \text { Binding }=\frac{[\text { Drug }]_{t}}{[\text { Drug }]_{t}+K_{d}}
$$

This equation captures the saturating S-shape of most dose-response curves that is characteristic of binary complex equilibria (Figure 1c). Critically, it identifies the dissociation constant $\left(K_{d}\right)$ as the most important parameter in drug design: changes in $K_{d}$ directly correlate with the drug's potency, or $\mathrm{EC}_{50}$ (effective concentration at which 50\% drug-target complex forms) (Figure 1c).

In classical pharmacokinetic theory, Michaelis-Menten kinetics is employed to model drugmetabolism and transport by hepatic and renal enzymes: ${ }^{15,16}$

$$
\text { velocity }=k_{\text {cat }}[\text { Enzyme }]_{t} \frac{[\text { Drug }]_{t}}{[\text { Drug }]_{t}+K_{m}}
$$

The history of each of these models and their influence on pharmacology is detailed in the supporting information to illustrate the parallels between classical pharmacological theory and ternary-complex theory discussed below. In addition, we present the derivations of these models in parallel to the derivations of ternary complex models to illustrate the overlaps in underlying mathematics as well.

\section{RATIONAL DESIGN OF MOLECULAR GLUES}

Rational design of Bifunctional therapies $(B)$ requires knowledge of the biological function of the two targets $(A \& C)$ to predict how they will behave when colocalized. Typically, one species is functionally Active $(\mathbf{A})$ and that activity is re-directed to the Contingent target (C) (Figure 1b). For PROTACs, the active species are E3 ubiquitin ligases and the contingent targets are oncogenic proteins. ${ }^{9}$ For many bsAbs, the active species are immune cells and the contingent targets are cancer cells. ${ }^{4}$ Conceptually, this redirection strategy is analogous to creating a new enzyme-substrate pair, or "re-wiring" the inputs of one pathway with the outputs of another.

Rational optimization of ternary therapeutics will be aided by an understanding of their pharmacodynamics and pharmacokinetics. While traditional drug-development might focus on improving a drug-target binding affinity $\left(K_{d}\right)$ to increase potency (Figure 1a,c; Eq. 1), ${ }^{12,17}$ ternary complex-based 
therapeutics are significantly more complicated. Ternary therapeutic pharmacodynamics feature multiple binding-affinities $\left(K_{A B}\right.$ and $\left.K_{B C}\right)$, multiple targets (A and $\left.C\right)$, and cooperative stabilizing or destabilizing interactions $(\alpha)$ between these two targets (Figure 1b). This additional complexity often results in a bellshaped dose-response curve for ternary-complex therapeutics (Figure 1d), distinguishing them from the S-shaped saturating behavior of most clinical drugs (Figure 1c). The ternary complex bell-shaped doseresponse curve has an intrinsic maximum effect dose $\left([B]_{t, \max }\right)$, and at this dose the fraction of possible ternary complex formed can be less than $100 \%$, which can limit the efficacy (y-axis magnitude) of bifunctional drugs (Figure 1d). ${ }^{18}$

\section{MANUSCRIPT SUMMARY}

Though current pharmacological theory is useful in the optimization of classical therapeutics, it is difficult to apply to the development of ternary therapeutics. For example, predicting how optimization of one of the two $K_{d}$ 's will affect efficacy and/or potency of a ternary therapeutic requires a new pharmacological framework. Here, we simplify and expand our 2013 models on ternary complex equilibria to generate a set of simple models to assist in the rational design of ternary complex therapeutics. ${ }^{18}$ By focusing on the maximum of the ternary complex curve $\left([B]_{t, \max }\right)$, we have derived a set of simple equilibrium and kinetic models that are analogous to the traditional pharmacological models discussed above (Eqs. 1 and 2). The behavior of these models hinges on the weaker of the two binding affinities, which has a disproportional effect on the amount of ternary complex that forms and the rate at which ternary complexes can redirect catalytic processes (Figure 1b). Finally, we have assembled a set of interactive online

a.

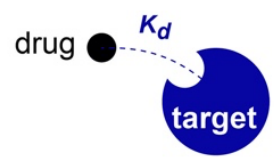

c.

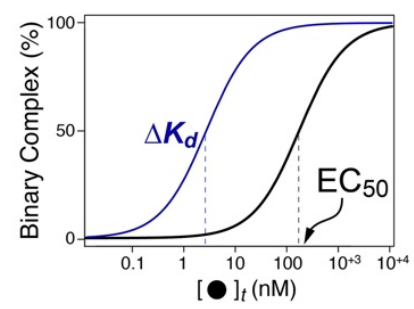

b.

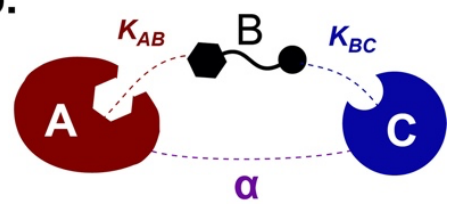

d.

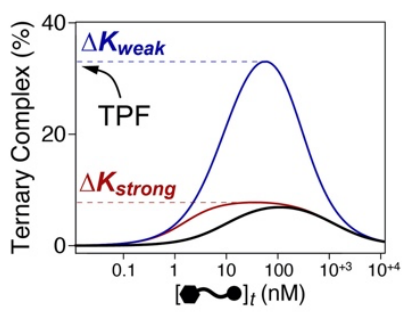

Figure 1. The dose-response curves of binary and ternary systems are distinct, and altering the $K_{d} s$ in these systems has different effects. a. Most clinical drugs bind and inhibit a singletarget protein. b. Ternary-complex drugs bind two targets simultaneously, creating a new PPI between A and C. c. Saturating dose-response curves for binary-complex drugs showing the effect of improving $K_{d}\left(\Delta K_{d}\right.$, blue). d. Bell-shaped dose-response curves for ternary-complex based drugs showing differential effects of improving the different binding constants.

tools to aid non-computational investigators in the application of ternary complex equilibrium and kinetic mathematics:

https://douglasslab.com/ternary equilibrium/

https://douglasslab.com/Btmax kinetics/ 


\section{Results and Discussion}

In 2013, we published the first exact equilibrium model for ternary complex equilibria. ${ }^{18}$ Though an important advance, this "Hill equation" for ternary complex equilibria was quite complex and has seen limited use outside of the computational community. Below we highlight relevant insights from that work and show how they can be expanded to understand the kinetic behavior of ternary complex drugs.

\section{TERNARY EQUILIBRIUM MAXIMA}

In our previous work, we identified the curve maximum as a uniquely solvable value:

$$
\frac{[A]_{t}+[C]_{t}+\frac{\left(\sqrt{K_{A B}}+\sqrt{K_{B C}}\right)^{2}}{\alpha}-\sqrt{\left([A]_{t}+[C]_{t}+\frac{\left(\sqrt{K_{A B}}+\sqrt{K_{B C}}\right)^{2}}{\alpha}\right)^{2}-4[A]_{t}[C]_{t}}}{2}
$$

where $[A]_{t}$ and $[C]_{t}$ reflect the total concentrations of $A$ and $C$, and $K_{A B}$ and $K_{B C}$ are the equilibrium dissociation constants of the bifunctional drug (B) for $A$ and $C$, respectively (Figure $1 \mathbf{b}$ ). In addition, $\alpha$ reflects cooperative interactions between $A$ and $C$, which can be stabilizing $(\alpha>1)$ or destabilizing $(\alpha<1)$. Using this equation, we showed that the maximum ternary complex formed often fails to saturate the receptors (A \& C) at any concentration of drug (B) (Figure 1d, max 10-40\%). This is a major conceptual difference between binary and ternary systems. In binary systems, high concentrations of ligand will completely saturate the target, but many ternary systems can only reach a fraction of their theoretical maximum.

To focus on the system parameters that have the greatest effect on the $[A B C]_{\max }$, we sought to simplify equation 3. As detailed in the supporting information, by assuming that one of the target concentrations in excess and one is limiting $\left([X]_{t}\right.$ and $\left.[L]_{t}\right)$, we can simplify equation 3 to:

$$
[A B C]_{\max } \approx[L]_{t} \frac{[X]_{t}}{[X]_{t}+\left(\sqrt{K_{A B}}+\sqrt{K_{B C}}\right)^{2} / \alpha}
$$

By assuming that one $K_{d}$ is 100 -fold greater than the other $\left(K_{\text {strong }} \& K_{\text {weak }}\right)$, we can further simplify equation 4 to:

$$
[A B C]_{\max } \approx[L]_{t} \frac{[X]_{t}}{[X]_{t}+K_{\text {weak }} / \alpha}
$$

Dividing both sides of equation 5 by the concentration of limiting reagent $\left([L]_{t}\right)$ we obtain an expression for the fraction of ternary complex that forms at the curve maximum:

$$
T P F=\frac{[X]_{t}}{[X]_{t}+K_{\text {weak }} / \alpha}
$$

We have previously defined this as the ternary partition fraction (TPF). The amount of ternary complex that can form is constrained by the limiting target $\left([L]_{t}\right)$. The TPF represents the fraction of the limiting target that is engaged in ternary complex at the optimum drug dose $\left([B]_{t, \max }\right)$. Equation 6 mirrors the Langmuir-Hill equation for binary complexes, and it reflects a similar saturation effect where excess terminal species $[X]_{t}$ can drive ternary complexes to saturate the limiting reagent $[L]_{t}$. Thus, the amount of ternary complex formed is largely a function of the parameters in equation 6 , so additional ternary complex will be obtained by: (1) increasing the excess receptor $\left([X]_{t}\right),(2)$ increasing the system's cooperativity $(\alpha)$, and $(3)$ decreasing/improving the weakest binding affinity $\left(K_{\text {weak }}\right)$. These predictions have been borne out in several experimental systems, as discussed below. 
Equation 6 defines the weakest binding affinity $\left(K_{\text {weak }}\right)$ as the most important binding parameter for increasing the fraction of ternary complex that forms at equilibrium. This effect can be observed directly with simulations from our 2013 model. Our model predicts that, in some systems, improving $K_{\text {weak }}$ can improve both the potency ( $x$-axis) and efficacy (y-axis) properties of a drug's dose-response curve, whereas improvements in $K_{\text {strong }}$ only improves the potency of the drug (Figure 1b). This prediction has been borne out experimentally from our work designing synthetic antibodies (Figure 2a). ${ }^{18,19}$ In addition, optimizing $K_{\text {weak }}$ has been noted by several other investigators to be critical to improving several ternary-complex therapies including: cytokines, heparin and antibody-based therapeutics. ${ }^{2,3,21-24}$

The $[X]_{t}$ term also has practical implications for experimental design. For example, if the TPF in an assay is low $(\sim 10 \%)$, it can be difficult to directly detect the ternary complex and/or the downstream reporters. If the levels of $A$ or $C$ can be adjusted in the assay, increasing the concentration of one can increase the TPF and thus the signal to noise ratio of the assay. We have leveraged this strategy in previous antibody recruiting work, where we were able to increase in vitro immune response by

\section{a. $\Delta K_{\text {weak }}\left(\Delta K_{F c R}\right)$ maximizes ternary complex}
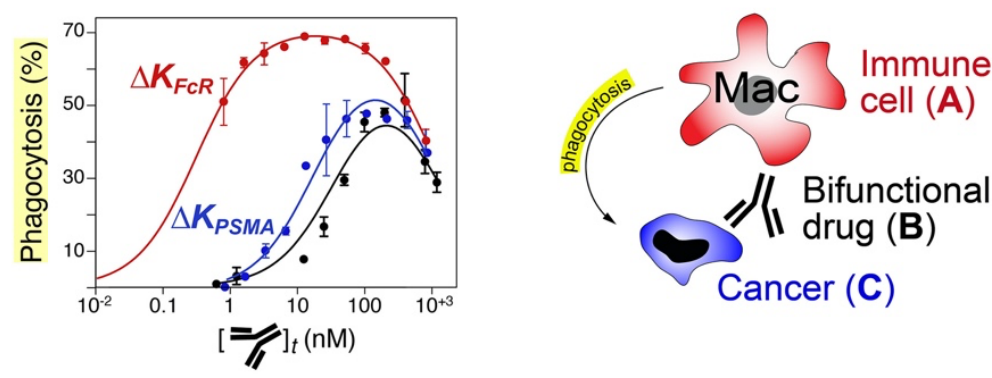

\section{b. $\Delta[\mathrm{X}]_{t}\left(\Delta[\mathrm{Ab}]_{t}\right)$ maximizes ternary complex}
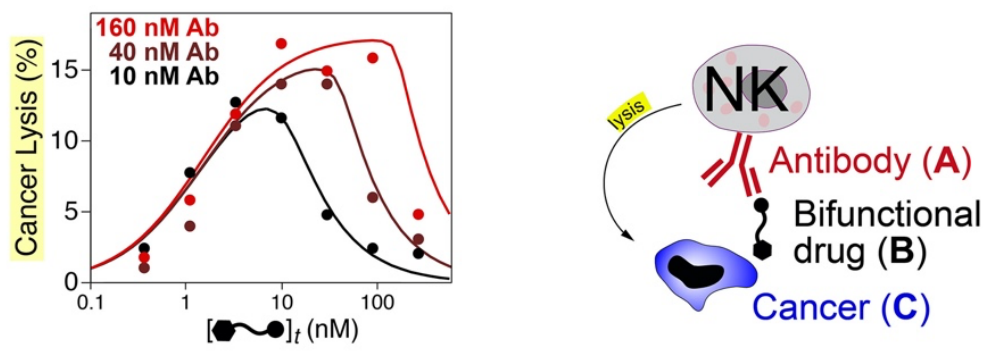

Figure 2. Two of the most important factors in increasing the ternary complex TPF are the weakest binding affinity $\left(K_{\text {weak }}\right)$ and the excess concentration $\left([\mathrm{X}]_{\mathrm{t}}\right)$. a. Previously published work $^{19}$ on small molecule antibody mimics has demonstrated that improvement in in the weaker binding affinity $\left(K_{A B}=K_{\text {weak }}\right)$ Fc-Receptor $(250 \mathrm{nM} \rightarrow 60 \mathrm{nM})$ had a larger effect on total phagocytosis than improving the stronger binding affinity (40 $\mathrm{nM} \rightarrow 20 \mathrm{nM}$ ) b. Previously published work $^{20}$ on antibody-recruiting molecules has demonstrated that increasing the concentration of the excess target species $\left([\mathrm{X}]_{\mathrm{t}}=\left([\text { Antibody }]_{\mathrm{t}}\right)\right.$ increases cancer cell lysis. increasing the concentration of Antibody $\left([X]_{t}\right.$ in that assay) (Figure $\left.\mathbf{2 b}\right){ }^{20}$

\section{TISSUE SELECTIVITY OF TERNARY COMPLEXES}

In addition, the $[X]_{t}$ term can be used to design tissue-specificity into ternary complex therapeutics by leveraging the differential expression of recruited targets in different tissues. With classical therapies, the fractional saturation of the target (or limiting reagent) will be the same across all tissues that the unbound drug can access (Equation 1). For ternary-based therapies, the fractional saturation of the limiting reagent will be controlled by the total concentration of the excess target in the tissue ([X]t, Eq. 6). Further, if one assumes non-saturating conditions $\left([\mathrm{X}]_{t}<K_{\text {weak }} / \alpha\right)$, then equation 6 enables one to make a simple estimate of the tissue-selectivity as the fraction of $[\mathrm{X}]_{t}$ in each tissue:

$$
\frac{T P F_{\text {lung }}}{T P F_{\text {kidneys }}}=\frac{[X]_{t}^{\text {lung }}}{[X]_{t}^{\text {kidneys }}}
$$

This ratiometric estimate of tissue selectivity is unitless and can therefore employ a diverse range of target-expression inputs (e.g., calibrated proteomics, RNAseq). For PROTACs, several recent publications have highlighted the differential tissue-expression of E3 ligases as a useful handle for engineering tissue-level specificity. ${ }^{9,25,26}$ 
For ternary systems in pre-equilibrium conditions, the equilibration of the complex occurs faster than the transformation by the enzyme $\left(k_{c a t}\right)$. As detailed in the supporting information, we estimate that most PROTAC systems can be approximated with the following pre-equilibrium analysis. Indeed, two recent kinetic analyses of PROTACs concluded that they were in a pre-equilibrium regime ${ }^{27,28}$. Assuming pre-equilibrium, the reaction velocity is the product of the $k_{\text {cat }}$ and the ternary complex concentration:

$$
\text { velocity }=k_{\text {cat }}[A B C]
$$

The maximum velocity of such a system occurs when the bispecific ligand is at $[\mathrm{B}]_{t, \max }$ and the amount of ternary complex is at its maximum height:

$$
V_{\text {max }}=k_{\text {cat }}[A B C]_{\text {max }}
$$

By substituting the $[A B C]_{\max }$ approximation from equation 5 , we obtain:

$$
V_{\text {max }}=k_{\text {cat }}[L]_{t} \frac{[X]_{t}}{[X]_{t}+K_{\text {weak }} / \alpha}
$$

Equation 10 has a similar form the classical Michaelis-Menten kinetic equation (Eq. 2), but

equation 10 is more general in that the enzyme can be either limiting $\left([\mathrm{L}]_{t}\right)$ or in excess $\left([\mathrm{X}]_{t}\right)$. As with the related TPF equation (Eq. 6), it is critical to remember that this equation does not model ternary-complex kinetics generally but rather ternary complex kinetics at the maximum of the doseresponse curve (Figure $\mathbf{3 b}$ ). Thus, this equation gives the maximum velocity and should be taken as a kinetic ceiling for ternary-complex based catalysis.

a. Pre-equilibrium ternary complex kinetics

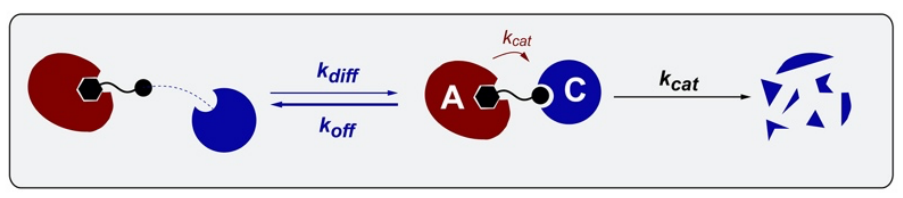

b. Initial rates ternary kinetics conceptual framework

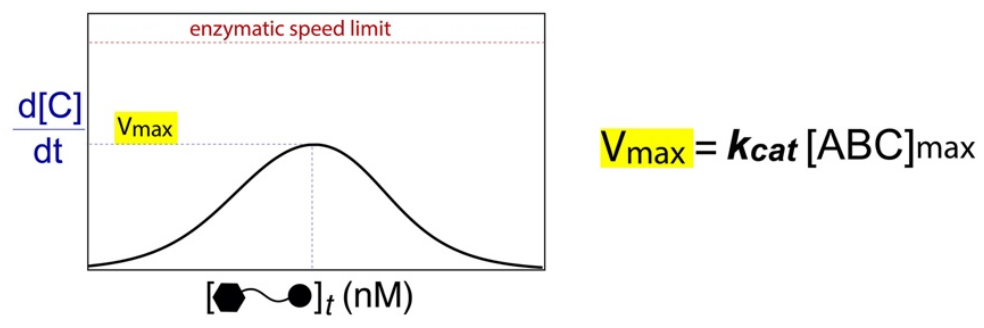

c. Pre-equilibrium timescales: conceptual framework

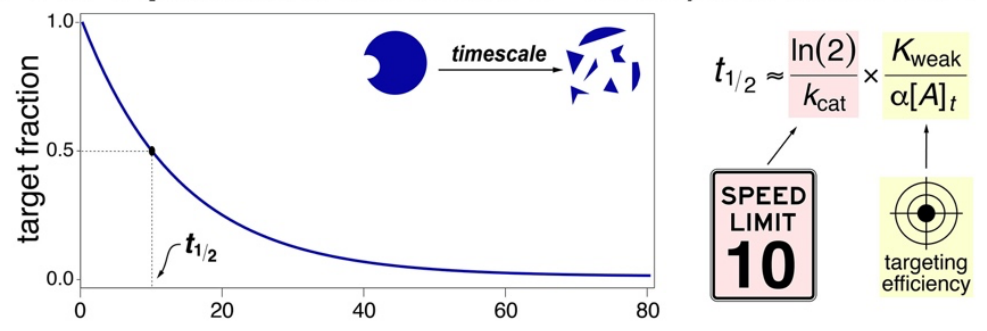

Figure 3. A pre-equilibirum framework for understanding the kinetics of PROTACS. a. Under pre-equilibrium conditions, the ternary complex equilibrates faster than catalysis. As detailed in the supporting information, we estimate that PROTAC systems where $K_{\text {weak }}$ is double-digit $\mathrm{nM}$ or higher should be in pre-equilibrium. For these analyses, we assume that $A$ is an E3 ligase, $B$ is the PROTAC, and $C$ is the degradation target. $\mathbf{b}$. In a ternary system, $V_{\max }$ occurs at $[\mathrm{B}]_{t, \max }$, as that is the ligand concentration where the ternary complex is greatest $\left([A B C]_{\max }\right)$. In many pre-equilibrium, ternary-complex systems, $V_{\max }$ will be lower than the enzymatic "speed limit", which is set by the maximum rate of the enzyme. c. As detailed in the supporting information, integration of the maximum ternary reaction velocity allows for estimation of the timescale on which the target (C) is transformed. This simple estimate is a product of the enzymatic "speed limit" and the targeting efficiency of the PROTAC.

Nevertheless, this equation provides a useful framework to understand the relative contributions of binding constant $\left(K_{\text {weak }}\right)$ and target concentrations $\left([L]_{t} \&[X]_{t}\right)$ to the kinetics of a ternary complex catalyzed process.

\section{PRE-EQUILIBRIUM TIMESCALES}

While the differential form of equation 10 can be useful for mathematical modeling, solving for the half-life of the target of degradation can provide an additional conceptual handle to understand the system. Fortunately, this can be obtained for equation 10 by integration with respect to the continent target $\mathrm{C}$. As the contingent target can be in excess or limiting, it is necessary to integrate equation 10 for both conditions, which is detailed in the supplemental information. Under non-saturating conditions ([X $]_{\mathrm{t}}$ $\left.<K_{\text {weak }} / \alpha\right)$ where the limiting species is not saturated, first order timescales are the same when $C$ or $A$ is in excess (Figure S10-11): 


$$
t_{1 / 2}^{C}=\frac{\ln (2)}{k_{\text {cat }}} \times \frac{K_{\text {weak }}}{\alpha[A]_{t}}
$$

where $[\mathrm{A}]_{\mathrm{t}}$ represents the active, or enzymatic, target. Under saturating conditions $\left([\mathrm{X}]_{\mathrm{t}}>K_{\text {weak }} / \alpha\right)$, the identity of the excess target gives different approximations. When the Active enzyme in in excess $\left([A]_{t}=\right.$ $[X]_{t}$ and $[A]_{t}>K_{\text {weak }} / \alpha$ ), the half-life of $C$ can be approximated as (Figure S11d):

$$
t_{1 / 2}^{c}=\frac{\ln (2)}{k_{\text {cat }}}
$$

Strikingly, equations 11-12 give a very simple conceptual framework for understanding the timescales of ternary-complex-mediated catalysis (Figure 2b). First, the kinetic "speed limit" can be directly inspected in the yellow shaded regions of equations 11-12. We call this the "speed limit" because the redirected degradation cannot be faster than the enzyme itself. Second, the targeting efficiency for these equations is highlighted in blue and is dependent on the expression level of the redirected enzyme (A) and the weakest binding affinity in the ternary complex $\left(K_{\text {weak }}\right)$.

\section{THERMODYNAMIC \& PHYSIOLOGICAL ESTIMATES OF MODEL PARAMETERS}

Thermodynamic and physiological estimates of parameters enable us to explore the behavior of the ternary complex models by assuming representative value ranges when exact values are not known. To generate these constraints, we collected data from several large databases. By curating $>60,000$ medicinal chemistry studies, the ChEMBL database has begun to define the "normal range" of drugtarget $K_{d} S$ as centered around 100 $\mathrm{nM}$ (Figure 4a). ${ }^{29}$ In addition, recent quantitative proteomics work has identified the average intracellular protein concentration as around $\sim 10 \mathrm{nM}$ (Figure 4a). . $^{30,31}$ These representative values are consistent with the assumption used to derive equation 11 above $\left([\mathrm{X}]_{t}<K_{\text {weak }} ; \sim 10 \mathrm{nM}<\sim 100 \mathrm{nM}\right)$. Taken together, these ranges indicate that Figure 1d gives an accurate picture of the equilibrium ternary complex achieved by most early molecular glue medicinal chemistry studies, with $K_{d} \mathrm{~S}$ between 10-1000 nM and most target concentrations between 1$100 \mathrm{nM}$. In addition to this thermodynamic data, several datasets on kinetic parameters exist as well. For example, the BRENDA database has catalogued over 33,000 enzymatic rate

a. Normal equilibrium values: $K_{d}$ and protein concentration


b. Normal kinetic values: $k_{c a t}$ and protein half-life
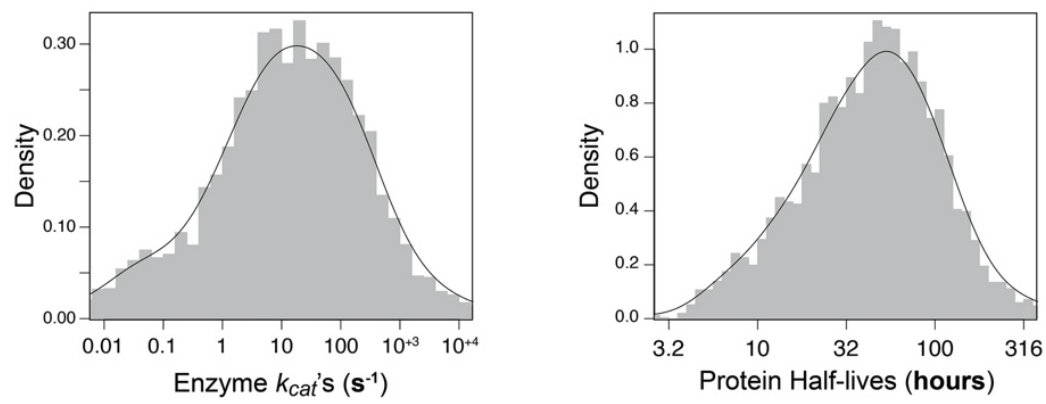

Figure 4. Reasonable ranges for parameter values can be estimated based on databases. a. Normal range of dissociation constants and protein concentrations compiled from medicinal chemistry studies and quantitative proteomics. b. Normal range of enzymatic rate constants and protein half-lives compiled from the biochemistry literature and quantitative proteomic study. constants $\left(\mathrm{k}_{\mathrm{cat}}\right)$, and the mean enzymatic turnover emerges as around 10x per second (Figure $\mathbf{4 b}$ ). ${ }^{32}$

By combining our pre-equilibrium model with literature values and typical physiological values, we can estimate the half-life of a PROTAC target. Typical saturated ubiquitination kinetics for E3 ligases occur on a 10 second time scale. Assuming the E3 ubiquitin ligase is expressed at $1 \mathrm{nM}$ and the PROTAC $K_{\text {weak }} \approx 1 \mu \mathrm{M}$, we would expect a ubiquitination time-scale on the order of approximately 3 hours. 


$$
t_{1 / 2}=\frac{\ln (2)}{k_{c a t}} \times \frac{K_{w e a k}}{\alpha \cdot[A]_{t}}=\sim 10 s \times \frac{1000 \mathrm{nM}}{1 \cdot 1 \mathrm{nM}}=\mathbf{1 0 , 0 0 0} \mathbf{s}=\mathbf{2 . 8} \mathbf{h r s}
$$

This half-life of hours is consistent with recent kinetic studies on PROTAC kinetics and is significantly faster than the typical rate of protein turnover ( 48 hours, Figure $\mathbf{4 b}$ ). ${ }^{27,28,30}$ Using equation 11 and representative physiological parameter ranges, we can see that a PROTAC with relatively weak binding affinities and low E3 ligase expression can still significantly accelerate the rate of target degradation.

\section{INTERACTIVE ONLINE APPLICATIONS}

We have presented the above simplifications (Eqs. 6, 10, 11) to emphasize the parameters that most affect the formation of ternary complex and cause subsequent enzymatic activity. For scientists who wish to model these ternary systems more completely, we have developed interactive online applications that calculate the full dose-response curve at equilibrium (https://douglasslab.com/ternary equilibrium/) and a time course for the enzymatic conversion of $\mathrm{C}$ when the bifunctional drug is at $[\mathrm{B}]_{t, \max }$ (https://douglasslab.com/Btmax kinetics/). Both of these applications use the exact equations for the simulations and show how our approximations for the critical points $\left(\mathrm{EC}_{50}, \mathrm{IC}_{50}, \mathrm{TPF}, t_{1 / 2}\right)$ fit onto these simulations (Figure S12). As the input values for the system change, the simple approximations displayed update to give the best approximation for the critical points. As discussed here and our previous work, ${ }^{18}$ we emphasize these simple approximations to show the parameters that most strongly affect the behavior of the critical points to enable more rational design of these systems, but we caution that there is always some error inherent in these approximations. To limit the error in the half-life estimate in the online application, we have employed:

$$
t_{1 / 2}=\frac{\ln (2)}{k_{c a t}} \times \frac{\left(\sqrt{K_{A B}}+\sqrt{K_{B C}}\right)^{2}}{\alpha[A]_{t}}
$$

rather than equation 11, as equation 15 gives less error when the $K_{d} s$ are within two orders of magnitude of each other.

For the online applications, we chose to place the system concentrations and $K_{d} S$ on the same logarithmic scales and in the same units to emphasize how their relative values affect the ternary system (Figure S12). We confined these values to reasonable ranges from the literature values (Figure S12). If an equilibrium dose-response curve modeled with different or more precise parameter inputs is needed, one can be obtained using the excel file in the supporting information of our previous paper. ${ }^{18}$ 


\section{Discussion}

We have adapted our previous ternary complex equilibrium analysis to model catalytic ternary complex therapeutics. We have shown that the most important parameters affecting the TPF also affect kinetic rates. Though we restricted our detailed analysis to the system's $V_{\max }$, increasing the TPF parameters is expected to improve enzymatic rates at all concentrations of bispecific drug (B). Thus, the most important parameters in ternary complex enzymatic rates are the excess concentration of receptor, the weaker $K_{d}$, and cooperativity between the two receptors (A and $C$ ).

Our more detailed kinetic analyses are restricted to the $V_{\max }$ of a pre-equilibrium system (Figure 3B). Thus, the estimated $V_{\max }$ and half-life will apply at $[B]_{t, \max }$, and represent a kinetic ceiling for the system. Additionally, we have assumed the system is in a pre-equilibrium state. As noted above, this was found to apply to several experimental PROTAC systems, and we estimate that many such systems can be assumed to be in pre-equilibrium. For this assumption, we have assumed that the $k_{\text {off, weak }} / \alpha>k_{\text {cat }}$, which would apply when $K_{\text {weak }}$ is double-digit $\mathrm{nM}$ or higher (assuming $k_{\text {on, weak }}=10^{7} \mathrm{M}^{-1} \cdot \mathrm{s}^{-1}, \alpha=1$, and $k_{\text {cat }}$ $\left.=0.1 \mathrm{~s}^{-1}\right)$. Of course, $k_{\text {on }}$ values for small molecules can range from $10^{6}-10^{9} \mathrm{M}^{-1} \cdot \mathrm{s}^{-1}$, some ternary systems may show cooperativity, and some enzymes of interest might be faster than typical E3 ligases $\left(\sim 0.1 \mathrm{~s}^{-1}\right)$. For systems where the two $k_{\text {off }} / \alpha$ values approach and become lower than $k_{\text {cat }}$, the system will be in steady state (Figure S9a). Such systems should operate close to the enzymatic "speed limits" shown in equations 12-13 or diffusion, whichever is limiting (Figure S9b).

Our derivation of the half-life timescales from the reaction velocity closely mirrors a similar derivation of classical Michaelis-Menten kinetics. Unfortunately, it seems that this integrated form of MM kinetics is not very well known, especially relative to its derivative form (Eq. 2). As detailed in our supporting information, half-life for binary systems can be estimated for subsaturated (Eq. D80) and saturated systems (Eq. D82), analogous to our equations 11-13. In fact, an exact solution to the halflife of a classical Michaelis-Menten process can be derived (Eq. D78). These derivations are described in detail in the supporting information.

We intend that these simple critical point estimates and more detailed interactive applications will enable the rational optimization of bispecific drugs that involve kinetic turnover. While numerical simulations of well-parameterized systems will provide more accurate simulations than our simplified models, we believe that analytical modeling is an important route to develop an intuition for how parameters will affect the behavior of complicated systems, even when detailed knowledge of the system parameters is lacking. We also hope that these models and interactive applications will be useful for scientists without significant computational training. 


\section{References}

1 Griffith, M. J. Kinetics of the Heparin-Enhanced Antithrombin-lii Thrombin Reaction - Evidence for a Template Model for the Mechanism of Action of Heparin. J Biol Chem 257, 7360-7365 (1982).

2 Streusand, V. J., Bjork, I., Gettins, P. G. W., Petitou, M. \& Olson, S. T. Mechanism of Acceleration of Antithrombin-Proteinase Reactions by Low-Affinity Heparin. J Biol Chem 270, 9043-9051, doi:DOI 10.1074/jbc.270.16.9043 (1995).

3 Carter, P. J. \& Lazar, G. A. Next generation antibody drugs: pursuit of the 'high-hanging fruit'. Nat Rev Drug Discov 17, 197-223, doi:10.1038/nrd.2017.227 (2018).

4 Labrijn, A. F., Janmaat, M. L., Reichert, J. M. \& Parren, P. Bispecific antibodies: a mechanistic review of the pipeline. Nat Rev Drug Discov 18, 585-608, doi:10.1038/s41573-019-0028-1 (2019).

5 Devos, A. M., Ultsch, M. \& Kossiakoff, A. A. Human Growth-Hormone and Extracellular Domain of Its Receptor - Crystal-Structure of the Complex. Science 255, 306-312, doi:DOI 10.1126/science.1549776 (1992).

6 Ilondo, M. M. et al. Receptor Dimerization Determines the Effects of Growth-Hormone in Primary Rat Adipocytes and Cultured Human Im-9 Lymphocytes. Endocrinology 134, 2397-2403, doi:DOI 10.1210/en.134.6.2397 (1994).

7 Schreiber, S. L. The Rise of Molecular Glues. Cell 184, 3-9, doi:10.1016/j.cell.2020.12.020 (2021).

8 Gao, H., Sun, X. \& Rao, Y. PROTAC Technology: Opportunities and Challenges. ACS Med Chem Lett 11, 237-240, doi:10.1021/acsmedchemlett.9b00597 (2020).

9 Samarasinghe, K. T. G. \& Crews, C. M. Targeted protein degradation: a promise for undruggable proteins. Cell Chem Biol, doi:10.1016/j.chembiol.2021.04.011 (2021).

10 Carter, P. J. \& Lazar, G. A. Next generation antibody drugs: pursuit of the 'high-hanging fruit'. Nature Reviews Drug Discovery 17, 197-223, doi:10.1038/nrd.2017.227 (2018).

11 Mullard, A. Targeted protein degraders crowd into the clinic. Nature Reviews Drug Discovery 20, 247-250 (2021).

12 Gesztelyi, R. et al. The Hill equation and the origin of quantitative pharmacology. Arch Hist Exact Sci 66, 427-438, doi:10.1007/s00407-012-0098-5 (2012).

13 Hill, A. V. The possible effects of the aggregation of the molecules of haemoglobin on its dissociation curves. Proceedings of the Physiological Society 40, 4-7 (1910).

14 Langmuir, I. The constitution and fundamental properties of solids and liquids Part I Solids. J Am Chem Soc 38, 2221-2295, doi:DOI 10.1021/ja02268a002 (1916).

15 Michaelis, L., Menten, M. L., Johnson, K. A. \& Goody, R. S. The original Michaelis constant: translation of the 1913 Michaelis-Menten paper. Biochemistry 50, 8264-8269, doi:10.1021/bi201284u (2011).

16 Michaelis, V. L. \& Menten, M. M. L. Die kinetik der invertinwirkung (The Kinetics of Invertase Action). Biochemische Zeitschrift 49, 333-369 (1913).

17 Kenakin, T. P. A pharmacology primer : theory, applications, and methods. 3rd edn, (Academic Press/Elsevier, 2009).

18 Douglass, E. F., Miller, C. J., Sparer, G., Shapiro, H. \& Spiegel, D. A. A Comprehensive Mathematical Model for Three-Body Binding Equilibria. J Am Chem Soc 135, 6092-6099, doi:10.1021/ja311795d (2013).

19 McEnaney, P. J. et al. Chemically Synthesized Molecules with the Targeting and Effector Functions of Antibodies. J Am Chem Soc 136, 18034-18043, doi:10.1021/ja509513c (2014). 
20 Murelli, R. P., Zhang, A. X., Michel, J., Jorgensen, W. L. \& Spiegel, D. A. Chemical Control over Immune Recognition: A Class of Antibody-Recruiting Small Molecules That Target Prostate Cancer. J Am Chem Soc 131, 17090-+, doi:10.1021/ja906844e (2009).

21 Junttila, I. S. et al. Redirecting cell-type specific cytokine responses with engineered interleukin4 superkines. Nat Chem Biol 8, 990-998, doi:10.1038/Nchembio.1096 (2012).

22 Jaks, E., Gavutis, M., Uze, G., Martal, J. \& Piehler, J. Differential receptor subunit affinities of Type I interferons govern differential signal activation. J Mol Biol 366, 525-539, doi:10.1016/j.jmb.2006.11.053 (2007).

23 Kalie, E., Jaitin, D. A., Podoplelova, Y., Piehler, J. \& Schreiber, G. The Stability of the Ternary Interferon-Receptor Complex Rather than the Affinity to the Individual Subunits Dictates Differential Biological Activities. J Biol Chem 283, 32925-32936, doi:10.1074/jbc.M806019200 (2008).

24 Desjarlais, J. R. \& Lazar, G. A. Modulation of antibody effector function. Exp Cell Res 317, 12781285, doi:10.1016/j.yexcr.2011.03.018 (2011).

25 Kannt, A. \& Dikic, I. Expanding the arsenal of E3 ubiquitin ligases for proximity-induced protein degradation. Cell Chem Biol, doi:10.1016/j.chembiol.2021.04.007 (2021).

26 Slabicki, M. et al. The CDK inhibitor CR8 acts as a molecular glue degrader that depletes cyclin K. Nature 585, 293-+, doi:10.1038/s41586-020-2374-x (2020).

27 Roy, M. J. et al. SPR-Measured Dissociation Kinetics of PROTAC Ternary Complexes Influence Target Degradation Rate. Acs Chem Biol 14, 361-368, doi:10.1021/acschembio.9b00092 (2019).

28 Riching, K. M. et al. Quantitative Live-Cell Kinetic Degradation and Mechanistic Profiling of PROTAC Mode of Action. Acs Chem Biol 13, 2758-2770, doi:10.1021/acschembio.8b00692 (2018).

29 Gaulton, A. et al. ChEMBL: a large-scale bioactivity database for drug discovery. Nucleic Acids Res 40, D1100-D1107, doi:10.1093/nar/gkr777 (2012).

30 Schwanhausser, B. et al. Global quantification of mammalian gene expression control. Nature 473, 337-342, doi:10.1038/nature10098 (2011).

31 Milo, R. \& Phillips, R. Cell biology by the numbers. (Garland Science, Taylor \& Francis Group, 2016).

32 Bar-Even, A. et al. The moderately efficient enzyme: evolutionary and physicochemical trends shaping enzyme parameters. Biochemistry 50, 4402-4410, doi:10.1021/bi2002289 (2011). 